\title{
Comparison of Contrast Agents for Atherosclerosis Imaging Using Cultured Macrophages: FDG Versus Ultrasmall Superparamagnetic Iron Oxide
}

\author{
Tomoko Satomi ${ }^{1}$, Mikako Ogawa ${ }^{2}$, Ikuo Mori ${ }^{3}$, Seigo Ishino ${ }^{3}$, Kazuki Kubo ${ }^{1}$, Yasuhiro Magata ${ }^{2}$, \\ and Tomoyuki Nishimoto ${ }^{1}$ \\ ${ }^{1}$ Metabolic Disease Drug Discovery Unit, Pharmaceutical Research Division, Takeda Pharmaceutical Company, Ltd., Fujisawa, \\ Japan; ${ }^{2}$ Medical Photonics Research Center, Hamamatsu University School of Medicine, Hamamatsu, Japan; and ${ }^{3}$ Biomolecular \\ Research Laboratories, Pharmaceutical Research Division, Takeda Pharmaceutical Company, Ltd., Fujisawa, Japan
}

\begin{abstract}
Various noninvasive imaging methods have been developed to evaluate atherosclerotic plaques. Among them, ${ }^{18} \mathrm{~F}-\mathrm{FDG}$ PET and MR imaging with ultrasmall superparamagnetic iron oxide particles (USPIO) have been used to quantify plaque inflammation. Both methods are based on the efficient uptake of FDG and USPIO by macrophages in atherosclerotic lesions. Differently polarized macrophages have been reported to have different characteristics that are involved in the pathologic development of atherosclerosis. M1 polarized macrophages are considered the more proatherogenic phenotype than M2 polarized macrophages. However, little is known regarding the association between macrophage polarization and FDG or USPIO accumulation. In this study, we investigated intracellular FDG and USPIO accumulation in M1 and M2 polarized macrophages. Methods: THP-1 macrophages were differentiated into M1 and M2 polarized macrophages. Under optimal glucose conditions, we investigated the ${ }^{3} \mathrm{H}$-labeled FDG uptake in $\mathrm{M} 1$ and $\mathrm{M} 2$ polarized macrophages. We then investigated intracellular USPIO uptake by M1 and M2 macrophages. Results: We found that M1 polarization, compared with M2 polarization, results in increased intracellular accumulation of FDG. To elucidate the mechanism by which FDG was preferentially accumulated in M1 macrophages, we examined messenger RNA expressions of glucose transporters (GLUTs) and hexokinases, which have pivotal roles in glucose uptake, and glucose-6-phosphatase (G6Pase), which catalyzes the reverse reaction of hexokinase. In M1 macrophages, GLUT-1, GLUT-3, hexokinase 1, and hexokinase 2 were upregulated and G6Pase was downregulated. In contrast to FDG, M1 polarization resulted in decreased intracellular accumulation of USPIO. We found that scavenger receptor A and CD11b, which are involved in USPIO binding and uptake, were significantly downregulated by M1 polarization. Conclusion: Compared with M2, proatherogenic M1 macrophages preferentially accumulated FDG but not USPIO, suggesting that FDG PET is a useful method for the detection of proinflammatory $\mathrm{M} 1$ macrophages.
\end{abstract}

\footnotetext{
Received Jun. 28, 2012; revision accepted Dec. 17, 2012.

For correspondence or reprints contact: Tomoko Satomi, Metabolic Disease Drug Discovery Unit, Takeda Pharmaceutical Company, Ltd., 26-1, Muraoka-Higashi 2-chome, Fujisawa, Kanagawa 251-8555, Japan.

E-mail: tomoko.satomi@takeda.com

Published online May 13, 2013.

COPYRIGHT @ 2013 by the Society of Nuclear Medicine and Molecular Imaging, Inc.
}

Key Words: atherosclerosis; FDG; USPIO; macrophage; polarization

J Nucl Med 2013; 54:999-1004

DOI: 10.2967/jnumed.112.110551

$\mathbf{T}$ he rupture of an atherosclerotic plaque heralds many cardiovascular complications such as myocardial and cerebral infarctions. Macrophage infiltration plays an important role in the development of vulnerable plaques. ${ }^{18} \mathrm{~F}$-FDG PET has been reported to be a promising tool for identifying vulnerable atherosclerotic plaques, because detection of these plaques depends on the extent of macrophage infiltration into the atherosclerotic lesions (1-3). To date, some clinical trials have been conducted to examine the usefulness of ${ }^{18} \mathrm{~F}-\mathrm{FDG}$ PET for evaluating atherosclerosis $(1,4)$. Furthermore, MR imaging with ultrasmall superparamagnetic iron oxide particles (USPIO) - which are also efficiently taken up by macrophages in the atherosclerotic lesion $(5,6)$-is used to quantify atherosclerotic plaques (6). Recently, macrophages have been considered as heterogeneous cells, which can be differently polarized in atherosclerotic plaques (7-9). Classically activated M1 macrophages promote the destabilization of atherosclerotic plaques, whereas alternatively activated M2 macrophages stimulate reparative processes, leading to stabilization of atherosclerotic plaques (10). Therefore, effective detection of M1 macrophages may help to predict cardiovascular events with greater accuracy. However, the type of macrophages that can be detected by ${ }^{18}$ F-FDG PET and USPIO MR imaging and the effect of macrophage polarization on FDG and USPIO accumulation are still unclear.

We report here the relationship between the macrophage polarization and imaging probe uptake to the cells. We first investigated the association between macrophage polarization and FDG and USPIO accumulation using human THP-1 macrophages. Then, to elucidate the molecular mechanism of FDG and USPIO accumulation in polarized macrophages, we analyzed the expression of genes responsible 
for ${ }^{18} \mathrm{~F}$-FDG uptake and metabolism and USPIO uptake and iron export.

\section{MATERIALS AND METHODS}

\section{Cell Culture and Chemicals}

Human THP-1 cells were obtained from Dainippon Sumitomo Pharma. Cell culture medium was purchased from Life Technologies. Fetal bovine serum was obtained from Thermo Fisher Scientific. Phorbol 12-myristate 13-acetate (PMA) was obtained from WAKO Pure Chemicals. Lipopolysaccharides (LPS) from Escherichia coli O111:B4 was obtained from Sigma-Aldrich. Recombinant human interferon- $\gamma$ (IFN $\gamma$ ), human interleukin (IL)-4, and human IL-13 were obtained from R\&D Systems. [5,6- $\left.{ }^{3} \mathrm{H}\right]$-2-fluoro-2-deoxyD-glucose $\left({ }^{3} \mathrm{H}-\mathrm{FDG}\right)$ was obtained from American Radiolabeled Chemicals. USPIO coated with alkali-treated dextran (average particle size, $28 \mathrm{~nm}$ ) was custom-synthesized by Meito Sangyo.

\section{Differentiation of THP-1 Cells into Macrophages and Polarization}

THP-1 cells were cultured in RPMI1640 supplemented with $10 \%$ heat-inactivated fetal bovine serum, penicillin $(100 \mathrm{U} / \mathrm{mL})$, and streptomycin $(100 \mu \mathrm{g} / \mathrm{mL})$. THP- 1 cells (seeded at $2 \times 10^{5}$ cells $/ \mathrm{cm}^{2}$ ) were differentiated into macrophages using $320 \mathrm{nM}$ PMA and polarized according to the method of Tjiu et al., with some modifications (11). For M1 polarization, cells were treated with PMA for $6 \mathrm{~h}$ and then cultured with PMA plus LPS $(10 \mathrm{ng} / \mathrm{mL})$ and IFN $\gamma(20 \mathrm{ng} / \mathrm{mL})$ for another $42 \mathrm{~h}$. For M2 polarization, cells were treated with PMA for $6 \mathrm{~h}$ and then cultured with PMA plus IL-4 $(20 \mathrm{ng} / \mathrm{mL})$ and IL-13 $(20 \mathrm{ng} / \mathrm{mL})$ for another $42 \mathrm{~h}$. We also developed control macrophages, which received no stimuli, that were differentiated from THP-1 cells by incubation with PMA for $48 \mathrm{~h}$. To evaluate the appropriate induction of polarization, we measured an M1 marker gene, inducible nitric oxide synthase (iNOS), and an M2 marker gene, mannose receptor $\mathrm{C}$ type 1 (MRC1).

\section{H-FDG Study}

Polarized macrophages were rinsed and then precultured in RPMI1640 containing $1 \mathrm{mM}$ glucose at $37^{\circ} \mathrm{C}$ for $1 \mathrm{~h}$. After the medium was replaced with fresh medium containing $1 \mathrm{mM}$ glucose and ${ }^{3} \mathrm{H}-\mathrm{FDG}(3.7 \mathrm{kBq} /$ well), cells were cultured for another $3 \mathrm{~h}$. Cells were washed twice with prechilled saline containing $25 \mathrm{mM}$ 4-(2-hydroxyethyl)-1-piperazineethanesulfonic acid (HEPES), $1 \mathrm{mM} \mathrm{CaCl}_{2}$, and $1 \mathrm{mM} \mathrm{MgCl}$ and dissolved with a $0.1 \%$ sodium dodecyl sulfate/0.1 M NaOH aqueous solution. The radioactivities of culture medium and cell lysates were measured using a liquid scintillation counter (Accu FLEX LSC 7400; Hitachi Aloka Medical, Ltd.). The cellular protein concentration was measured using the DC Protein Assay Kit (Bio-Rad). ${ }^{3} \mathrm{H}-\mathrm{FDG}$ accumulation was expressed as radioactivities in cell lysate per milligram of cell protein.

\section{USPIO Study}

Macrophages were incubated with USPIO (400 $\mu \mathrm{g} \mathrm{Fe} / \mathrm{mL})$ for the last $16 \mathrm{~h}$ of the 2 - $\mathrm{d}$ treatment with polarizing stimulus at $37^{\circ} \mathrm{C}$ in $5 \% \mathrm{CO}_{2}$. The cells were washed twice with prechilled saline containing $25 \mathrm{mM}$ HEPES, $1 \mathrm{mM} \mathrm{CaCl}_{2}$, and $1 \mathrm{mM} \mathrm{MgCl}_{2}$ and were dissolved with $0.5 \%$ Triton $\mathrm{X}-100$ (MP Biomedicals) aqueous solution. The iron contents of cell lysates were measured using the Iron Assay Kit (BioChain). Cellular protein concentration was measured using the DC Protein Assay Kit. The USPIO accumulation was expressed as cellular iron content per milligram of cell protein.

\section{Quantitative Real-Time Polymerase Chain Reaction}

Cellular total RNA was extracted using the RNeasy mini kit (Qiagen) and converted into complementary DNA using the High Capacity cDNA Reverse Transcription Kit (Life Technologies). Gene expression was analyzed using the 7900HT Fast Real-Time PCR System (Life Technologies) with the TaqMan Universal Master Mix II (Life Technologies) and primer-probe sets of TaqMan Gene Expression Assays (Life Technologies) for the following genes: iNOS (Hs00167248_m1), MRC1 (Hs00267207_ $\mathrm{m} 1$ ), scavenger receptor A (SR-A; Hs00234012_m1), glucose transporter (GLUT)-1 Hs00892681_m1), GLUT-3 (Hs00359840_ $\mathrm{m} 1$ ), hexokinase1 (HK1; Hs00175976_m1), hexokinase2 (HK2; Hs00606086_m1), glucose-6-phosphatase (G6Pase; Hs00292720_ m1), CD11b (Hs00355885_m1), CD36 (Hs00354519_m1), ferroportin 1 (Fpn1; Hs00205888_m1), and ferritin light chain (FTL; Hs00830226_gH). $\beta$-actin (Hs99999903_m1) was used as an endogenous control gene. Relative messenger RNA (mRNA) expression was calculated using the $\Delta \Delta \mathrm{Ct}$ method.

\section{Statistics}

Data are presented as mean $\pm \mathrm{SD}$. Statistical analyses were performed using SAS software (version 8.2; SAS Institute, Inc.). Either a Tukey parametric multiple-comparison test or a Steel-Dwass nonparametric multiple-comparison test (Bartlett test; $P<0.05$ ) was used for comparisons among the 3 different macrophage types (i.e., M1, M2, and control). $P$ values of less than 0.05 were considered statistically significant.

\section{RESULTS}

\section{Macrophage Polarization}

Macrophages treated with IFN $\gamma$ and LPS showed significant upregulation of iNOS (Fig. 1A) $(P<0.05)$, whereas those treated with IL-4/IL-13 showed significant upregulation of MRC1 (Fig. 1B) $(P<0.05)$. The M1 marker genes CCR7 and IL-1 $\beta$ were also significantly upregulated in macrophages treated with IFN $\gamma /$ LPS, and the M2 marker gene IL-1 ra was significantly upregulated in macrophages treated with IL-4/IL-13 (data not shown). Hence, we used these macrophages as for M1 polarization and M2 polarization for the following experiments.

\section{${ }^{3} \mathrm{H}-F D G$ Accumulation in M1 and M2 Macrophages}

To study accumulation of FDG in macrophages, we first optimized the glucose concentration in the medium, because FDG PET signals decrease if blood glucose levels exceed 200 $\mathrm{mg} / \mathrm{dL}$. In a preliminary study using a medium containing a high glucose concentration of $200 \mathrm{mg} / \mathrm{dL}$, FDG accumulation in macrophages was markedly reduced (data not shown). In a low-glucose medium of $1.8 \mathrm{mg} / \mathrm{dL} \quad(\sim 0.1$ $\mathrm{mM}$ ), we observed that cells were damaged (data not shown). Therefore, we added glucose at $1 \mathrm{mM}$ to the medium-an amount sufficient to detect FDG accumulation without damaging the cells. M1 macrophages, compared with M2 macrophages, showed a 2.6-fold increased uptake of ${ }^{3} \mathrm{H}-\mathrm{FDG}$ (Fig. 2) $(P<0.01)$.

\section{Expression of Glucose Metabolism-Related Genes in Macrophages}

We measured the mRNA expression of glucose metabolism-related genes to elucidate the mechanism by which 


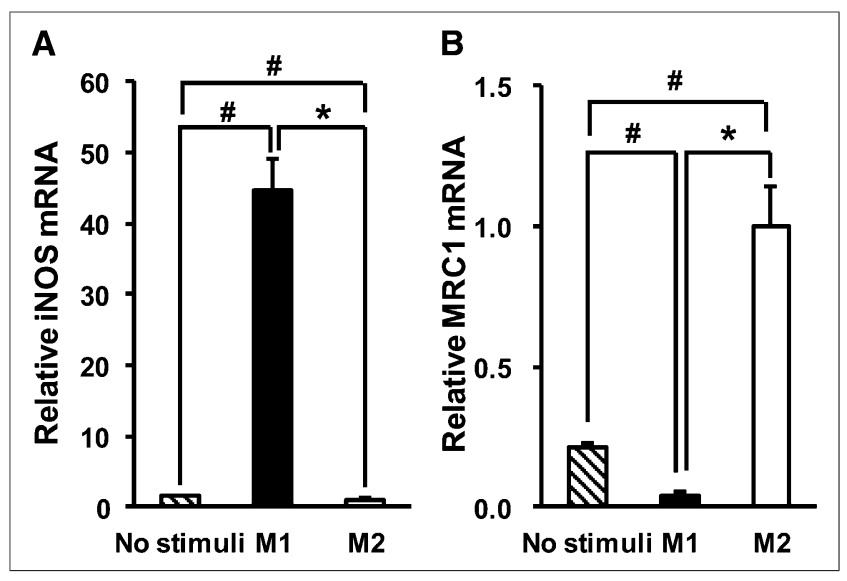

FIGURE 1. Gene expression of $M 1$ and $M 2$ markers in human THP-1 macrophages treated with each polarizing factor: iNOS as M1 marker (A) and MRC1 as M2 marker (B). Data are presented as mean \pm SD $(n=6)$. ${ }^{*} P<0.05$, comparison between M1 and M2. ${ }^{\#} P<0.05$, compared with no stimuli by Steel-Dwass test.

FDG was preferentially taken up by M1 macrophages. We found that GLUT-1 and GLUT-3, which are major isoforms of a glucose transporter in macrophages $(12,13)$, were significantly upregulated in M1 macrophages, compared with M2 macrophages (Fig. 3) $(P<0.01$ and $<0.01$, respectively). HK1 and HK2, which catalyze the intracellular phosphorylation of glucose into glucose-6-phosphate, were also significantly upregulated in M1 macrophages, compared with M2 macrophages (Fig. 3) $(P<0.01$ and $<0.01$, respectively). However, the expression of G6Pase, which is an enzyme that catalyzes the reverse reaction of hexokinases, was significantly downregulated in M1 macrophages, compared with M2 macrophages (Fig. 3) $(P<0.01)$. These results suggest that the increase of FDG accumulation in M1 macrophages was caused by the upregulation of GLUT and hexokinase genes as well as the downregulation of G6Pase gene.

\section{USPIO Accumulation in M1 and M2 Macrophages}

Previous USPIO accumulation studies have used macrophages treated with USPIO in concentrations ranging from 50 to $500 \mu \mathrm{g} \mathrm{Fe} / \mathrm{mL}$ and for time periods lasting from 1 to $72 \mathrm{~h}$ (14-17). On the basis of our preliminary study findings, macrophages were incubated with USPIO $(400 \mu \mathrm{g} \mathrm{Fe} / \mathrm{mL})$ for the last $16 \mathrm{~h}$ of the $2-\mathrm{d}$ treatment with polarizing stimulus at $37^{\circ} \mathrm{C}$

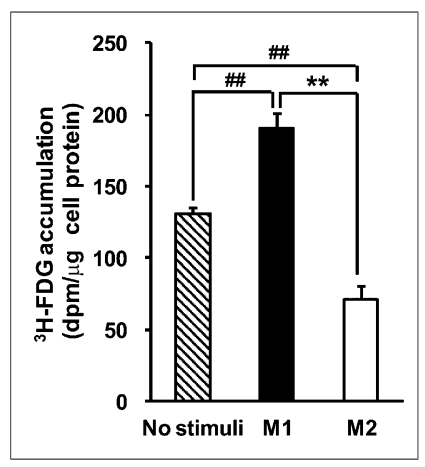

in $5 \% \mathrm{CO}_{2}$. USPIO accumulation in the cells increased linearly with both USPIO concentration and incubation time (data not shown). In contrast to FDG, USPIO accumulation in M2 macrophages was 1.4-fold higher than that in M1 macrophages (Fig. 4) $(P<0.01)$.

\section{Expression of USPIO Uptake- and Iron Export- Related Genes in Macrophages}

To elucidate the mechanism by which USPIO was preferentially taken up by M2 macrophages, compared with M1 macrophages, we measured the mRNA expression of SR-A and CD11b $(14,18)$. Both SR-A and CD11b were significantly upregulated in M2 macrophages, compared with M1 macrophages (Fig. 5) $(P<0.01$ and $<0.01$, respectively). Considering that other scavenger receptors may contribute to the uptake of USPIO, we measured the expression of CD36, another major scavenger receptor. As shown in Figure 5, CD36 was also upregulated in M2 macrophages. The expression of Fpn1, the major iron exporter in mammalian cells (19), was also significantly upregulated in M2 macrophages, compared with M1 macrophages (Fig. 5) $(P<0.01)$. There was no significant difference in the expression of FTL, which is responsible for intracellular iron storage (20), between M1 and M2 macrophages (Fig. 5).

\section{DISCUSSION}

${ }^{18}$ F-FDG PET and USPIO MR imaging have been clinically used to quantify atherosclerotic plaques, because both FDG and USPIO are efficiently taken up by macrophages in atherosclerotic lesions. Recently, macrophages in varying polarized states have been reported to have different characteristics that are involved in the pathologic development of atherosclerosis $(7,10)$. In this study, we compared the ability of differently polarized macrophages to take up FDG and USPIO using human THP-1 macrophages. We showed that FDG was taken up preferentially by M1 macrophages, whereas USPIO was taken up by M2 macrophages.

The preferential uptake of FDG by M1 macrophages led to the hypothesis that M1 and M2 differed in the expression of the glucose uptake-related molecules because FDG is taken up by cells in a manner analogous to glucose. We observed that the mRNA expression of GLUT-1 and GLUT-3 was upregulated in M1 macrophages. Kim et al. also reported that the mRNA expression of GLUT-1 was upregulated and intracellular FDG accumulation increased in mouse RAW264.7 macrophages stimulated by LPS (21). In addition, we showed that the mRNA expression of HK1 and HK2, which are major hexokinase isoforms, was upregulated, whereas the mRNA expression of G6Pase was downregulated in M1 macrophages. FDG is taken up into cells via glucose transporters and then phosphorylated by hexokinases to FDG-6-phosphate (FDG6P) in a mechanism similar to glucose. FDG6P is retained inside the cell, such that it can neither follow the process of glycolysis nor easily pass through the plasma membrane. If G6Pase, the catalyst of the reverse reaction of hexokinase, converts FDG6P into FDG, FDG can 
FIGURE 3. Expression of glucose metabolism-related genes in human THP-1 macrophages: GLUT-1, GLUT-3, HK1, HK2, and G6Pase. Data are presented as mean \pm SD $(n=6) .{ }^{\star \star} P<0.01$, comparison between M1

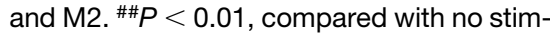
uli by Tukey test.
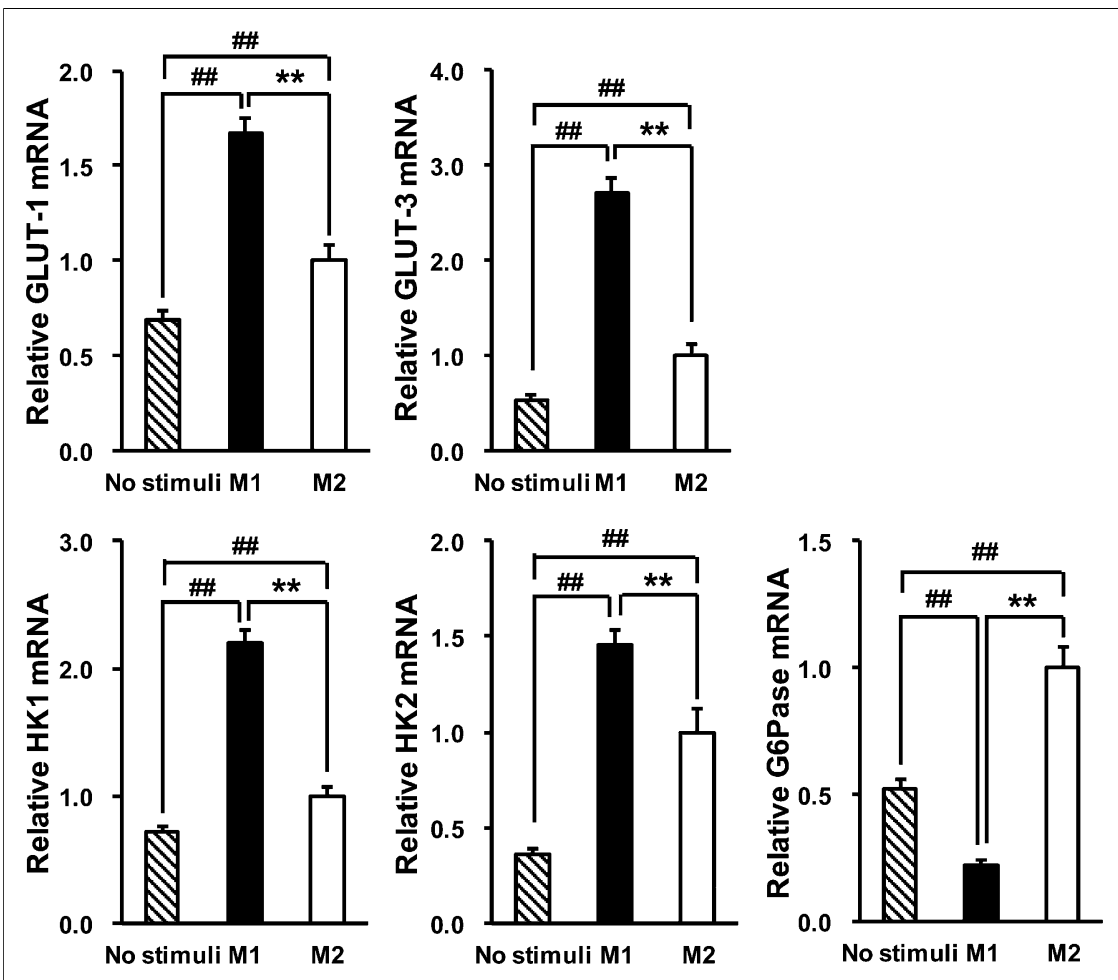

theoretically diffuse out of cells via glucose transporters. The present study suggests that the regulation of gene expression of key molecules involved in glucose uptake contributed to the increase in intracellular FDG accumulation in M1 macrophages. In particular, the increased intracellular FDG uptake via glucose transporters, the phosphorylation of FDG by hexokinases, and the decrease in dephosphorylation of FDG6P by G6Pase contributed to the increase of FDG uptake in M1 macrophages. On the other hand, inconsistent with our observation, Folco et al. reported that inflammatory stimulation did not increase the glucose uptake in human primary monocytes (22). They used IFN $\gamma$ or a combination of IFN $\gamma, \mathrm{TNF} \alpha$, and IL1 $\beta$ as inflammatory stimulation, whereas we adopted a classic M1 stimulation for characterizing typical phenotypes of M1 macrophages. We need to consider a more relevant stimulation to atherosclerosis in future experiments.

FIGURE 4. Intracellular USPIO accumulation in human THP-1 macrophages. Data are presented as mean $\pm \operatorname{SD}(n=6)$. ${ }^{\star \star} P<0.01$, comparison between M1 and M2. ${ }^{\#} P<0.01$, compared with no stimuli by Tukey test.
Small iron oxide particles (SPIO) and USPIO have been used clinically as superparamagnetic MR imaging contrast agents. These iron oxide particles are efficiently taken up by macrophages. A range of molecules is considered to be involved in SPIO and USPIO uptake and iron export. Lunov et al. identified the clathrin-mediated SR-A-dependent endocytosis as a major pathway of USPIO uptake in peripheral blood monocyte-derived macrophages (14). von Zur Muhlen et al. showed that USPIO uptake was inhibited by treatment with anti-CD11b antibody in PMA-stimulated human peripheral blood monocytes (18). In the present study, we determined that both SR-A and CD11b were significantly upregulated in M2 macrophages, which might contribute to the increase in USPIO uptake by M2 macrophages (Fig. 5). $\mathrm{Gu}$ et al. reported that internalized USPIO in mouse macrophages was degraded in lysosomes, and free iron was released into intracellular iron pools (23). Fpn1 is known to have an important role in the export of iron from cells (19). We observed that Fpn1 was also upregulated in M2 macrophages, compared with M1 macrophages (Fig. 5), consistent with the previous report (24). In the present study, we observed a statistically significant increase of USPIO uptake in M2 macrophages, compared with M1 macrophages, but the difference was relatively small, despite the marked induction of genes related to the uptake of USPIO. The upregulation of Fpn1 could cause the reduction of the cellular iron levels, which might attenuate the accumulation of USPIO in M2 macrophages. Taken together, the accumulation of cellular USPIO was higher in M2 macrophages than in M1 macrophages, most likely as a result of the balance of transcriptional 

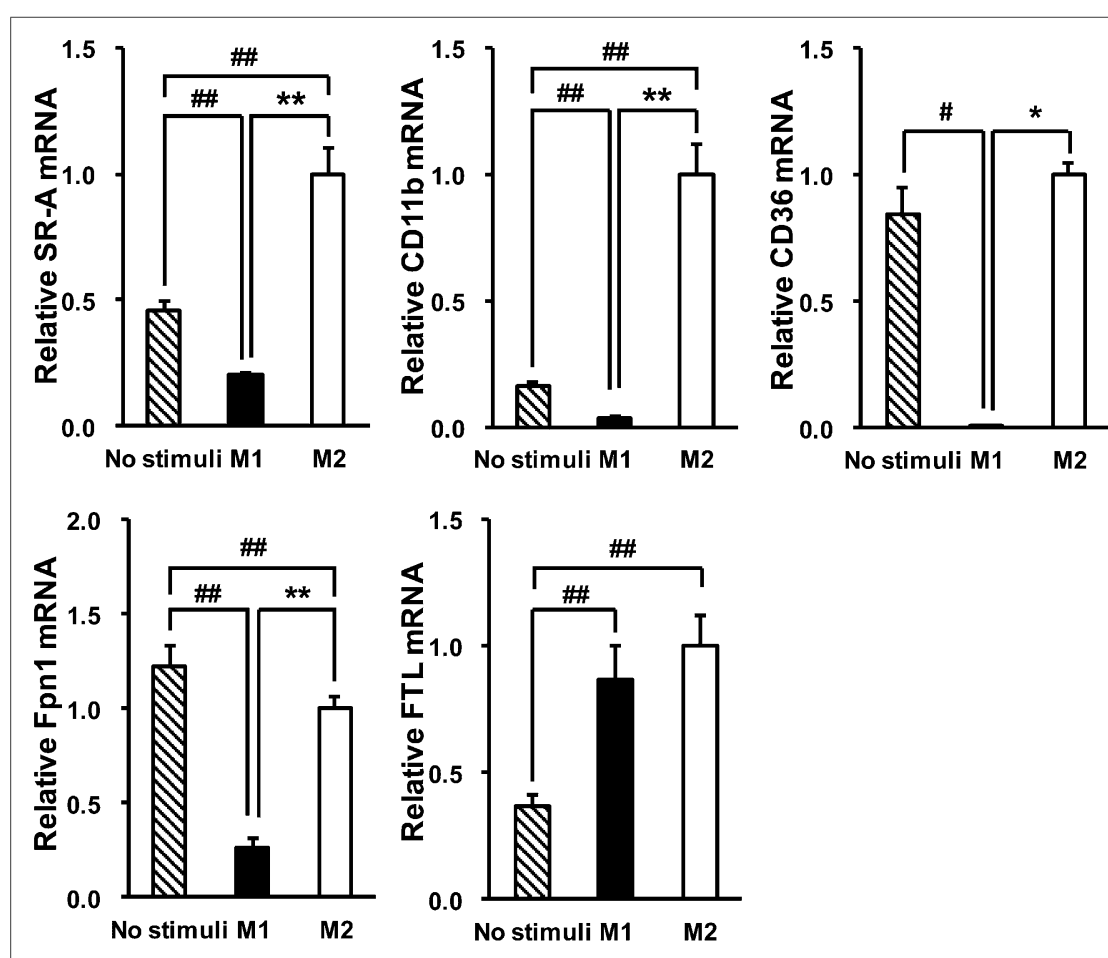

FIGURE 5. Expression of USPIO uptakeand iron export-related genes in human THP-1 macrophages: SR-A, CD11b, CD36, Fpn1, and FTL. Data are presented as mean $\pm \operatorname{SD}(n=6) .{ }^{*} P<0.05$ and ${ }^{* *} P<0.01$, comparison between $\mathrm{M} 1$ and $\mathrm{M} 2$. ${ }^{\#}<<$ 0.05 and ${ }^{\# \# P}<0.01$, compared with no stimuli by Tukey test or Steel-Dwass test. regulation of genes related to uptake and export of USPIO. In the present study, there were no difference in USPIO accumulation between M2 macrophages and control ones (Fig. 4), suggesting the difficulty of visualizing only M2 macrophages in distinction from nonpolarized macrophages by USPIO. Recently, it has been reported that the status of macrophage polarization in atherosclerotic plaques was dramatically changed in the progression and regression process of atherosclerosis using an experimental murine model $(8,25)$. In addition, a substantial number of macrophages were polarized in those lesions. Therefore, USPIO should be able to light up the mainly M2 lesion, although we need to pay attention to the false-positive results. In contrast to our findings, Rogers et al. reported that not only Th2 cytokine IL4 but also Th1 cytokine IFNy increased SPIO uptake in mouse J774A.1 macrophages (26). However, differing experimental conditions including cell type and polarization methods might explain these discrepancies. Rogers et al. used IFN $\gamma$ and IL-4 alone as Th1 and Th 2 cytokines, respectively, whereas we used IFN $\gamma$ in addition to LPS and IL-4 plus IL13 as M1-polarizing and M2-polarizing stimuli, respectively. In this study, we investigated the USPIO uptake with a 16-h incubation to obtain the reasonably sufficient amount of intracellular USPIO for quantitative analysis, whereas the incubation time was $3 \mathrm{~h}$ for FDG. The exposure time might affect the uptake of FDG and USPIO in M1 and M2 polarized macrophages. Cell viability also should be another important factor to evaluate the cellar uptake. In this study, at least, the cell damage was not observed morphologically. Further investigation about exposure time and cell viability is needed for precise analysis.
The ultimate purpose of atherosclerosis imaging, and the detection of vulnerable rupture-prone plaques in particular, is the accurate prediction of cardiovascular events. Recently, studies have suggested that the quality rather than quantity of macrophages is critical in the evolution of vulnerable plaques (27-29). M1 macrophages play a key role in the development of atherosclerosis, which is mediated by production of proinflammatory cytokines or reactive nitrogen oxides, whereas M2 macrophages have antiatherogenic properties mediated by the production of antiinflammatory cytokines and the suppression of proinflammatory signaling $(30,31)$. However, the contribution of these differently polarized macrophages to vulnerable plaque formation, potentially resulting in plaque rupture, has not yet been fully understood. For example, Mauriello et al. recently reported that M2 but not M1 macrophages were present in the fibrous cap near the rupture site in the human carotid artery, suggesting that M2 macrophages might also modulate the process of plaque rupture (32). Although further investigations regarding the roles of M1 and M2 macrophages in atherosclerotic lesions are needed, it is clear that macrophage polarization affects plaque diagnosis and prognosis. In our preliminary in vivo study, stronger signals were observed in abdominal plaques than in plaques in the aortic arch in en face autoradiography of the whole aorta of ${ }^{14} \mathrm{C}-\mathrm{FDG}$-injected apolipoprotein E knockout mice (Supplemental Fig. 1; supplemental materials are available online only at http://jnm.snmjournals.org). The M1 markers iNOS, IL-1 $\beta$, and CCR7 were upregulated in the abdominal plaques, whereas the M2 marker MRC1 was upregulated in the aortic arch plaques (Supplemental Fig. 2). These results suggest that ${ }^{18}$ F-FDG PET should dominantly visualize the 
M1 macrophage-infiltrated area. Some reports have suggested that ${ }^{18}$ F-FDG PET may be useful to monitor the therapeutic effects of drugs (33-35), and some of the drugs are reported to affect the polarization of macrophages $(8,36)$. Because therapies that reduce M1 macrophages are garnering more attention, it becomes increasingly important to select appropriate imaging methods that accurately detect plaque development.

\section{CONCLUSION}

The present study provides in vitro evidence that ${ }^{18} \mathrm{~F}-\mathrm{FDG}$ PET preferentially detects proinflammatory M1 macrophages, whereas USPIO MR imaging preferentially detects antiinflammatory M2 macrophages. Although further in vivo investigations are needed, ${ }^{18}$ F-FDG PET imaging should be useful in the detection of proinflammatory M1 macrophages.

\section{DISCLOSURE}

The costs of publication of this article were defrayed in part by the payment of page charges. Therefore, and solely to indicate this fact, this article is hereby marked "advertisement" in accordance with 18 USC section 1734. No potential conflict of interest relevant to this article was reported.

\section{ACKNOWLEDGMENTS}

We thank Drs. Masaaki Mori, Masakuni Noda, Shota Ikeda, Richard Urquhart, Ryogo Hayashi, Hiroki Nagase, Aya Tanaka, Takahisa Matsuda, and Yukio Yamada in our laboratories for their invaluable suggestions and feedback.

\section{REFERENCES}

1. Tawakol A, Migrino RQ, Bashian GG, et al. In vivo ${ }^{18} \mathrm{~F}$-fluorodeoxyglucose positron emission tomography imaging provides a noninvasive measure of carotid plaque inflammation in patients. $J$ Am Coll Cardiol. 2006;48:1818-1824.

2. Ogawa M, Ishino S, Mukai T, et al. ${ }^{18} \mathrm{~F}-\mathrm{FDG}$ accumulation in atherosclerotic plaques: immunohistochemical and PET imaging study. J Nucl Med. 2004;45:1245-1250.

3. Zhang Z, Machac J, Helft G, et al. Non-invasive imaging of atherosclerotic plaque macrophage in a rabbit model with F-18 FDG PET: a histopathological correlation [abstract]. BMC Nucl Med. 2006;6:3.

4. Tahara N, Imaizumi T, Virmani R, Narula J. Clinical feasibility of molecular imaging of plaque inflammation in atherosclerosis. J Nucl Med. 2009;50:331-334.

5. Trivedi RA, Mallawarachi C, U-King-Im JM, et al. Identifying inflamed carotid plaques using in vivo USPIO-enhanced MR imaging to label plaque macrophages. Arterioscler Thromb Vasc Biol. 2006;26:1601-1606.

6. Tang TY, Howarth SP, Miller SR, et al. The ATHEROMA (Atorvastatin Therapy: Effects on Reduction of Macrophage Activity) Study. Evaluation using ultrasmall superparamagnetic iron oxide-enhanced magnetic resonance imaging in carotid disease. J Am Coll Cardiol. 2009;53:2039-2050.

7. Khallou-Laschet J, Varthaman A, Fornasa G, et al. Macrophage plasticity in experimental atherosclerosis. PLOS ONE. 2010;5:e8852.

8. Feig JE, Parathath S, Rong JX, et al. Reversal of hyperlipidemia with a genetic switch favorably affects the content and inflammatory state of macrophages in atherosclerotic plaques. Circulation. 2011;123:989-998.

9. Bouhlel MA, Derudas B, Rigamonti E, et al. PPARgamma activation primes human monocytes into alternative M2 macrophages with anti-inflammatory properties. Cell Metab. 2007;6:137-143.

10. Mantovani A, Garlanda C, Locati M. Macrophage diversity and polarization in atherosclerosis: a question of balance. Arterioscler Thromb Vasc Biol. 2009;29:1419-1423.

11. Tjiu JW, Chen JS, Shun CT, et al. Tumor-associated macrophage-induced invasion and angiogenesis of human basal cell carcinoma cells by cyclooxygenase2 induction. J Invest Dermatol. 2009;129:1016-1025.

12. Fu Y, Maianu L, Melbert BR, Garvey WT. Facilitative glucose transporter gene expression in human lymphocytes, monocytes, and macrophages: a role for
GLUT isoforms 1,3 , and 5 in the immune response and foam cell formation. Blood Cells Mol Dis. 2004;32:182-190.

13. Ahmed N, Kansara M, Berridge MV. Acute regulation of glucose transport in a monocyte-macrophage cell line: Glut-3 affinity for glucose is enhanced during the respiratory burst. Biochem J. 1997;327:369-375.

14. Lunov O, Zablotskii V, Syrovets T, et al. Modeling receptor-mediated endocytosis of polymer-functionalized iron oxide nanoparticles by human macrophages. Biomaterials. 2011;32:547-555.

15. Müller K, Skepper JN, Tang TY, et al. Atorvastatin and uptake of ultrasmall superparamagnetic iron oxide nanoparticles (Ferumoxtran-10) in human monocytemacrophages: implications for magnetic resonance imaging. Biomaterials. 2008;29: 2656-2662.

16. Yancy AD, Olzinski AR, Hu TC, et al. Differential uptake of ferumoxtran-10 and ferumoxytol, ultrasmall superparamagnetic iron oxide contrast agents in rabbit: critical determinants of atherosclerotic plaque labeling. J Magn Reson Imaging. 2005;21:432-442.

17. Raynal I, Prigent P, Peyramaure S, Najid A, Rebuzzi C, Corot C. Macrophage endocytosis of superparamagnetic iron oxide nanoparticles: mechanisms and comparison of ferumoxides and ferumoxtran-10. Invest Radiol. 2004;39: 56-63.

18. von Zur Muhlen C, von Elverfeldt D, Bassler N, et al. Superparamagnetic iron oxide binding and uptake as imaged by magnetic resonance is mediated by the integrin receptor Mac-1 (CD11b/CD18): implications on imaging of atherosclerotic plaques. Atherosclerosis. 2007;193:102-111.

19. Donovan A, Lima CA, Pinkus JL, et al. The iron exporter ferroportin/Slc40a1 is essential for iron homeostasis. Cell Metab. 2005;1:191-200.

20. Torti FM, Torti SV. Regulation of ferritin genes and protein. Blood. 2002;99: 3505-3516.

21. Kim C, Kim S. Taurine chloramine inhibits LPS-induced glucose uptake and glucose transporter 1 expression in RAW 264.7 macropages. Adv Exp Med Biol. 2009;643:473-480.

22. Folco EJ, Sheikine Y, Rocha VZ, et al. Hypoxia but not inflammation augments glucose uptake in human macrophages: implications for imaging atherosclerosis with ${ }^{18}$ fluorine-labeled 2-deoxy-D-glucose positron emission tomography. $\mathrm{J} \mathrm{Am}$ Coll Cardiol. 2011;58:603-614.

23. Gu J, Xu H, Han Y, et al. The internalization pathway, metabolic fate and biological effect of superparamagnetic iron oxide nanoparticles in the macrophagelike RAW264.7 cell. Sci China Life Sci. 2011;54:793-805.

24. Recalcati S, Locati M, Marini A, et al. Differential regulation of iron homeostasis during human macrophage polarized activation. Eur J Immunol. 2010;40:824-835.

25. Feig JE, Rong JX, Shamir R, et al. HDL promotes rapid atherosclerosis regression in mice and alters inflammatory properties of plaque monocyte-derived cells. Proc Natl Acad Sci USA. 2011;108:7166-7171.

26. Rogers WJ, Basu P. Factors regulating macrophage endocytosis of nanoparticles: implications for targeted magnetic resonance plaque imaging. Atherosclerosis. 2005; 178:67-73.

27. Hartung D, Schäfers M, Fujimoto S, et al. Targeting of matrix metalloproteinase activation for noninvasive detection of vulnerable atherosclerotic lesions. Eur J Nucl Med Mol Imaging. 2007;34(suppl):S1-S8.

28. Matter CM, Wyss MT, Meier P, et al. ${ }^{18} \mathrm{~F}$-choline images murine atherosclerotic plaques ex vivo. Arterioscler Thromb Vasc Biol. 2006;26:584-589.

29. Jarrett BR, Correa C, Ma KL, Louie AY. In vivo mapping of vascular inflammation using multimodal imaging. PLOS ONE. 2010;5:e13254.

30. Gordon S, Taylor PR. Monocyte and macrophage heterogeneity. Nat Rev Immunol. 2005;5:953-964.

31. Wolfs IM, Donners MM, de Winther MP. Differentiation factors and cytokines in the atherosclerotic plaque micro-environment as a trigger for macrophage polarisation. Thromb Haemost. 2011;106:763-771.

32. Mauriello A, Servadei F, Sangiorgi G, et al. Asymptomatic carotid plaque rupture with unexpected thrombosis over a non-canonical vulnerable lesion. Atherosclerosis. 2011;218:356-362.

33. Fayad ZA, Mani V, Woodward M, et al. Safety and efficacy of dalcetrapib on atherosclerotic disease using novel non-invasive multimodality imaging (dalPLAQUE): a randomised clinical trial. Lancet. 2011;378:1547-1559.

34. Tahara N, Kai H, Ishibashi M, et al. Simvastatin attenuates plaque inflammation: evaluation by fluorodeoxyglucose positron emission tomography. J Am Coll Cardiol. 2006;48:1825-1831.

35. Ogawa M, Magata Y, Kato T, et al. Application of ${ }^{18} \mathrm{~F}-\mathrm{FDG}$ PET for monitoring the therapeutic effect of antiinflammatory drugs on stabilization of vulnerable atherosclerotic plaques. J Nucl Med. 2006;47:1845-1850.

36. Fujita E, Shimizu A, Masuda Y, et al. Statin attenuates experimental antiglomerular basement membrane glomerulonephritis together with the augmentation of alternatively activated macrophages. Am J Pathol. 2010;177: 1143-1154. 